\title{
Correlation Between the Optical Performance of the Reflective Polarizer and the Structure of LCD Backlight
}

\author{
Mi-Yeon Yu, Byung-Woo Lee, Jeong Ho Lee, and Jae-Hyeon Ko* \\ Department of Physics, Hallym University, Chuncheon 200-702, Korea
}

(Received February 17, 2009 : accepted March 16, 2009)

\begin{abstract}
The optical performance of the reflective polarizer was investigated over three kinds of direct-lit backlights and/or different configurations of the optical sheets. The optical gain factor achieved by using the reflective polarizer increased as the diffuse nature of the optical sheet was enhanced, because of the more efficient polarization conversion. In addition, the gain factor decreased substantially in flat-lamp backlights compared to tubular-lamp backlights, which was ascribed to the high loss factor during the polarization recycling process due to the complex structure and large area of flat lamps. All these results suggested that reducing the loss factor of the backlight is very important for efficient polarization conversion and thus high optical gain of the reflective polarizer.
\end{abstract}

Keywords: Backlight unit (BLU), Reflective polarizer, Gain factor, Loss factor, Liquid crystal display (LCD)

OCIS codes : (120.2040) Displays; (150.2950) Illumination; (230.3720) Liquid-crystal devices

\section{INTRODUCTION}

A backlight unit (BLU) is a two-dimensional illumination device used for the non-emissive liquid crystal display (LCD). BLU supplies the LCD panel with bright, homogeneous white light by use of light sources and optical components. Light sources are used to generate visible light having an appropriate spectrum. Many light sources have been adopted or under development for BLU, such as cold-cathode fluorescent lamps (CCFL), external-electrode fluorescent lamps (EEFL), flat fluorescent lamps (FFL), light emitting diodes (LED), etc [1]. Since these are normally point or one-dimensional light sources, several optical sheets are necessary in order to achieve a collimated, homogeneous illumination condition on BLU. A diffuser plate(DP), a diffuser sheet(DS), a prism sheet, and a reflective polarizer are typical representative optical sheets used in the BLU. DP and DS are mainly used for diffusing the visible light while the prism film and the reflective polarizer are used for enhancing the brightness on the LCD panel.

The most typical prism sheet consists of one-dimensional prism grooves of which the cross-section is an isosceles

*Corresponding author: hwangko@hallym.ac.kr triangle with an apex angle of 90 degrees. Light rays diffusely incident onto the bottom flat surface of the prism sheet are collimated toward the on-axis direction via refraction on the surface of the prism grooves. If the rays do not meet the condition of refraction for collimation, they are reflected back toward the bottom part of BLU for "angle recycling". That is, their angles are changed via diffuse reflection on several components comprising the bottom part of BLU, and some of them are returned back to the prism sheet, are refracted at the prism surface toward the normal direction and contribute to the on-axis luminance [2]. The operating principle of the reflective polarizer is similar to that of the prism sheet. The polarization component of the light emitted from BLU orthogonal to the transmission axis of the bottom polarizer of LCD is reflected back at the reflective polarizer for the "polarization recycling" process. This undesired polarization component is partly transformed into the desired one which can transmit through the bottom polarizer of LCD while it is diffusely reflected on other optical sheets or light sources. In this case, efficient polarization conversion at the bottom part of BLU is very important for obtaining high luminous efficacy of BLU and LCD.

Since the overall device performances of BLU are 
determined by the combination of the light sources and the optical sheets, it is very important to optimize the optical matching condition between these two essential components. In particular, the structure of BLU should be optimized from the view point of efficient recycling processes for the brightness enhancement films since the optical gain in the on-axis luminance in terms of the prism sheet or the reflective polarizer is highly dependent on the structure of the light sources, as well as on the detailed configuration of optical sheets. Although the operating principle of the multi-layer reflective polarizer has been reported in detail [3], there has been no detailed report on the correlation between the optical performance of the reflective polarizer and the structure of BLU except for our previous study [4]. There are other reports on the optimization of combinations of optical sheets [5] or the quantification of luminance properties on each optical sheet in BLU [6]. The purpose of this paper is to report the quantitative correlation between the on-axis luminance gain by the reflective polarizer and the detailed structure of LCD backlights, such as light sources and the configuration of optical sheets.

\section{EXPERIMENT}

Three kinds of different light sources and corresponding BLU's have been used for the present study. The first two are 32-inch CCFL and $\mathrm{Hg}$-type 28-channel FFL BLU's. The last one is 6-inch Xe-type FFL BLU. The detailed description of these BLUs can be found in our previous studies $[7,8]$. In order to investigate the effect of light sources and the configuration of optical sheets on the optical gains by the reflective polarizer, conventional optical sheets have been put over the light sources for luminance measurements, such as a diffuser plate(DP), a diffuser sheet(DS), a one-dimensional prism film (BEF II from 3M, abbreviated as BEF), and a reflective polarizer (DBEF-D from 3M, abbreviated as DBEF). A new diffuser sheet consisting of hemi-spherical microlenses monolithically integrated on a flat substrate (UTE II film from MNTECH, abbreviated as UTE) was also used in the Xe-type FFL BLU.

In order to examine the polarization state of the transmitted light, an absorptive polarizer was put on the 32-in CCFL BLU, and the luminance on each sheet included in this BLU was measured as the transmission axis of the polarizer was rotated by 5 or 10 degrees. In addition, in order to extract the quantitative gain factor by the reflective polarizer, the absorptive polarizer whose trans mission axis is parallel to that of the DBEF was inserted between the BLU and the luminance colorimeter, and the luminance was measured on various combinations of optical films with and without DBEF. The angular distribution of the luminance on each film, as well as the on-axis luminance, has been measured by using either the EZ - contrast of ELDIM or the luminance colorimeter (BM-7, TOPCON). In order to find out the final luminance gain on the LCD panel, the on-axis luminance on each configuration of optical sheets has been obtained on the "BLU+panel" combination. Commercially-available 32-inch LCD TV (Samsung Electronics) was used for this measurement.

\section{RESULTS}

\section{Polarization states on the optical sheets}

Figure 1 shows the dependence of the luminance on the angle of the transmission axis of the absorptive polarizer on each film in CCFL BLU. In this figure, the angle of 0 degrees refers to the condition that the transmission axis of the absorptive polarizer is horizontal, i.e., parallel to the direction of the prism grooves as well as to the transmission axis of DBEF. As is expected, the transmitted light through DP (and DS) is completely unpolarized due to the diffuse scattering of incident rays via randomly-dispersed beads included in the structure of these films. The transmitted light through the onedimensional prism sheet is slightly polarized by about $8 \%$, i.e., the luminance of the vertically-polarized light is higher than that of the horizontally-polarized light. This result can be understood by considering the transmission and reflection properties of each polarization state of the light incident on the prism surfaces. According to the Fresnel's law [9], the overall reflectance of the $\mathrm{s}$-polarization, of which the polarization direction is parallel to the prism grooves, is higher than that of the orthogonal $\mathrm{p}$-polarization. It should thus be pointed out that the polarization state on the prism sheet should

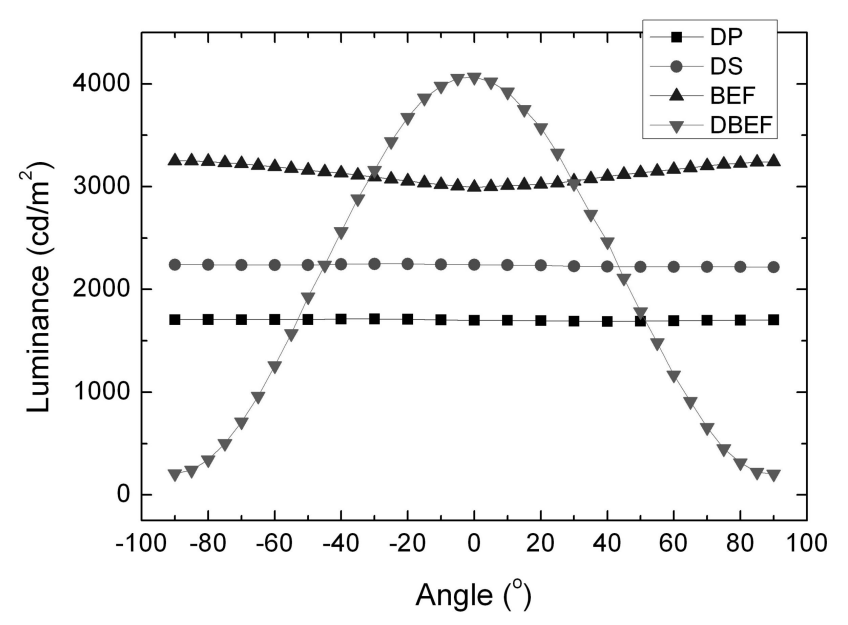

FIG. 1. The dependence of the luminance on the angle of the transmission axis of the absorptive polarizer on each film in CCFL BLU. The angle of 0 degrees refers to the condition that the transmission axis of the polarizer is horizontal, i.e., parallel to the direction of the prism grooves as well as to the transmission axis of DBEF. 
be considered in the optimization of the overall performances of backlights. On the reflective polarizer (DBEF), the transmitted light is strongly polarized along the horizontal direction which is the transmission axis of DBEF. The extinction ratio is about 20 .

\section{A simple model on the gain process of the reflective polarizer}

In order to estimate the performance of the reflective polarizer and its correlation with the structure of light sources or optical sheets, a simple theoretical model on the gain process of the reflective polarizer was used according to Ref. [10]. In this model, the backlight is divided into two parts, the reflective polarizer and the other components, such as other optical sheets, light sources, reflection sheet, etc., which will be mentioned as a conversion element. The light power incident on the reflective polarizer from below is composed of the power of a desired light with a horizontally-polarized state $\left(P_{H}\right)$ and that of an undesired one with a verticallypolarized state $\left(P_{V}\right) . P_{H}$ is transmitted through while $P_{V}$ is reflected from the reflective polarizer. When $P_{V}$ is reflected again on the lower part of BLU, it is either converted into the desired state, or remains in the same polarization state, or disappears via some loss processes. The power fractions of these three light components with respected to the total downward light power will be denoted as $c, s$, and $L_{c}$, respectively [10]. $c+s+L_{c}=1$ according to the energy conservation. The gain process of the reflective polarizer can be described by a series of transmission, reflection, conversion, and loss processes as is shown in Figure 2. In this approach, it is assumed that $P_{H}$ is transmitted through while $P_{V}$ is reflected from the reflective polarizer by $100 \%$, i.e., any possible light loss during the transmission or the reflection at DBEF is neglected. The cumulative light power transmitted through DBEF may easily be obtained as follows [10].

$$
\begin{aligned}
P_{\text {total }} & =P_{H}+c P_{V}+c P_{V} s+c P_{V} s^{2}+c P_{V} s^{3}+\cdots \\
& =P_{H}\left[1+\frac{c\left(P_{V} / P_{H}\right)}{1-s}\right]
\end{aligned}
$$

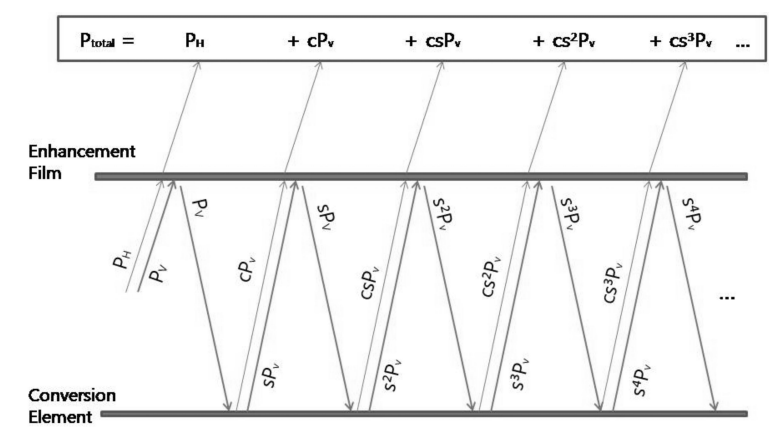

FIG. 2. A schematic diagram for explaining the polarization recycling process of the reflective polarizer. Regarding the notations, see the text.
The optical gain factor $G$ by the reflective polarizer is defined by the ratio of the total transmitted power on DBEF to the power of the light of the same polarization without DBEF, that is, $P_{H}$. Thus,

$$
G=\frac{P_{\text {total }}}{P_{H}}=1+\frac{c\left(P_{V} / P_{H}\right)}{1-s}
$$

Since the conversion element includes diffuse reflector, DS and DP, it is natural to assume that $c=s$. Therefore, the loss factor of the conversion element $L_{c}$ can be determined if the power ratio of both polarization states on the BLU without DBEF $\left(P_{V} / P_{H}\right)$ and the gain factor $G$ are measured by experiments. The equation for $L_{c}$ is given by Eq. (3).

$$
L_{c}=1-2 c==\frac{P_{V} / P_{H}-G+1}{P_{V} / P_{H}+G-1}
$$

\section{Correlation between the gain factor and the struc- ture of BLU's}

As a first step, the gain factor of DBEF was examined on several combinations of optical sheets in CCFL BLU. For this purpose, an absorptive polarizer of which the transmission axis was parallel to that of DBEF was put over the BLU and the luminance was measured first without and then with DBEF at the same combination of optical sheets. The results are summarized in Table 1. On DP where the emitting light distribution is almost Lambertian, the gain factor $G$ amounts to 1.78 , the largest among the three configurations. The corresponding loss factor $L_{c}$ becomes the smallest. If additional optical sheets such as DS or BEF are added sequentially on DP, $G$ decreases while $L_{c}$ increases. It indicates that the optical performance of the reflective polarizer is very sensitive to the detailed configuration of the optical sheets included in the BLU.

Second, the gain factor of DBEF was investigated on an LCD panel combined by several kinds of BLU having different light sources but the same combination of optical films. The tested light sources were CCFL, Hg-type FFL, and $\mathrm{Xe}$-type FFL backlights. The results are summarized in Table 2, from which two major characteristics can be derived. First, the gain factor of DBEF is very sensitive to what kind of light source is adopted in BLU even if the same combination of optical sheets is used. $G$ of CCFL BLU is larger than those of $\mathrm{Hg}$-type and Xe-type FFL BLU's. In particular, the loss factors of Xe-type BLU are much larger than the other two BLU's. Second, $G$ becomes larger when BEF is placed by DS by 10 18\% depending on the light source. This is consistent with the result of the first set of experiments where $G$ becomes larger when BEF is removed from BLU. 
TABLE 1. Luminance values on an absorptive polarizer which was put on the CCFL BLU at three combinations of optical films measured without and with DBEF. The transmission axis of the absorptive polarizer was set to be parallel to that of $\mathrm{DBEF}$. The gain factor, $P_{V} / P_{H}$, and the loss factor $L_{c}$ of each combination are also shown.

\begin{tabular}{c|c|c|c|c|c}
\hline \hline $\begin{array}{c}\text { Combination of } \\
\text { optical films }\end{array}$ & $\begin{array}{c}\text { Luminance without } \\
\text { DBEF }\left(\mathrm{cd} / \mathrm{m}^{2}\right)\end{array}$ & $\begin{array}{c}\text { Luminance with } \\
\text { DBEF }\left(\mathrm{cd} / \mathrm{m}^{2}\right)\end{array}$ & Gain Factor $G$ & $P_{V} / P_{H}$ & Loss Factor $L_{c}$ \\
\hline DP & 1700 & 3020 & 1.78 & 1.00 & 0.12 \\
\hline DP+DS & 2200 & 3420 & 1.55 & 1.00 & 0.29 \\
\hline DP+DS+BEF & 3100 & 3990 & 1.29 & 1.08 & 0.58 \\
\hline
\end{tabular}

TABLE 2. Luminance values on the LCD panel with two combinations of optical films measured without and with DBEF for three kinds of backlights. The gain factor and the loss factor $L_{c}$ of each combination are also shown. The same $P_{V} / P_{H}$ in Table 1 was used to calculate the loss factor.

\begin{tabular}{c|c|c|c|c|c}
\hline \hline \multirow{2}{*}{ Backlight } & $\begin{array}{c}\text { Combination of } \\
\text { optical films }\end{array}$ & $\begin{array}{c}\text { Panel Luminance } \\
\text { without DBEF }\left(\mathrm{cd} / \mathrm{m}^{2}\right)\end{array}$ & $\begin{array}{c}\text { Panel Luminance with } \\
\text { DBEF }\left(\mathrm{cd} / \mathrm{m}^{2}\right)\end{array}$ & Gain Factor G & Loss Factor $L_{c}$ \\
\hline \multirow{2}{*}{ CCFL BLU } & DP+DS+BEF & 577 & 750 & 1.30 & 0.57 \\
\cline { 2 - 6 } & DP+DS+DS & 417 & 644 & 1.54 & 0.30 \\
\hline Hg-type & DP+DS+BEF & 473 & 597 & 1.26 & 0.61 \\
\cline { 2 - 6 } FFL BLU & DP+DS+DS & 377 & 520 & 1.04 & 0.45 \\
\hline Xe-type & Lamp+DS & 763 & 793 & 1.12 & 0.79 \\
\cline { 2 - 6 } FFL BLU & Lamp+UTE & 726 & 816 & & 0.92 \\
\hline
\end{tabular}

\section{DISCUSSION AND CONCLUSION}

The present study revealed that the optical gain factor, i.e., the enhancement factor of the on-axis luminance, by the reflective polarizer is determined not only by the structure of the light source but also by the configuration of optical sheets adopted in BLU. The optical performance of the reflective polarizer is affected by the polarization conversion process during the diffuse reflection on other optical components in BLU such as optical sheets, light sources, or reflectors. There are thus two conditions for obtaining high gain factor of the reflective polarizer: (1) the polarization state of the downward rays should be changed effectively on the conversion element; (2) the loss factor of the conversion element should be small.

In the case of CCFL BLU, there are 16 tubular lamps (outer diameter: $3 \mathrm{~mm}$ ) below which a diffuse reflector is located. Since the area of these light sources is much smaller than the total area of BLU, the diffuse reflector will mainly contribute to the polarization conversion process for the downward rays from DBEF and other optical sheets. The high reflectivity as well as the diffuse nature of this film is expected to exhibit a low loss factor, a high conversion efficiency for the light of the undesired polarization and thus a high gain factor, as has been demonstrated in this study. However, the FFL, which is a one-body light source, covers all the area of BLU in contrast to the case of tubular-lamp-based BLU's. FFL is usually composed of many layers including two glass substrates, one or two phosphor layers, one reflection layer, and/or sheet electrodes or a protection layer. Downward rays will come in contact with these layers, be reflected, scattered or absorbed resulting in lower reflectivity compared to that of diffuse reflector in CCFL BLU [4]. In particular, the upper surface of the Xe-type FFL is flat in contrast to the curved multi-channel structure of $\mathrm{Hg}$-type $\mathrm{FFL}$, and thus the rays will experience multiple reflections between the FFL and other optical sheets more often, during which the rays will get more chance to be absorbed or scattered [8]. This seems to be the main origin of the high loss factors observed from the Xe-type FFL BLU's.

The fact that the gain factor of $\mathrm{DBEF}$ is higher in the BEF-free BLU than in the BEF-based BLU, which can be seen from Table 2, may be understood in the same context. The surface of the prism sheet is specular, while those of DS and DP are diffuse in nature and thus tend to change the angle and the polarization state of incident rays more effectively than $\mathrm{BEF}$. In this respect, the efficiency of polarization conversion via which the undesired polarization state is transformed into the desired state is expected to become higher in the BEF-free BLU than in the BEF-based BLU. Similarly, DP may be considered as a more effective conversion component than DS from the view point of polarization recycling since it exhibits a much higher gain factor.

In summary, the dependence of the gain factor of the reflective polarizer on the detailed structure of BLU, such as light sources and the combination of optical 
sheets, was examined in detail for the first time, and the loss factor of the conversion element was derived. The optical gain factor becomes higher in CCFL BLU and lower in FFL BLU's, indicating that the loss factor of the conversion element increases in FFL backlights due to the much larger surface area, the multi-layer structures and thus the higher possibility of absorption and scattering compared to tubular lamp-based backlights. In addition, the optical gain factor increases as the diffuse nature of the optical sheet is enhanced, because the conversion efficiency of the undesired polarization state into the desired one becomes higher during the random scattering process in DP and DS than the reflection/transmission on BEF. All these results clearly suggest that the correlation between the optical performance of the brightness enhancement films and the backlight structure should be considered in the optimization of the backlight structure.

\section{ACKNOWLEDGMENT}

The authors highly appreciate scientists and engineers at the Samsung Corning Co. for supplying us with FFL backlights in addition to kind support on the experiment. This work was supported by the New University for Regional Innovation (NURI) project by the Korean Ministry of Education and Human Resources Development $\left(5^{\text {th }}\right.$ year project in 2008 2009).

\section{REFERENCES}

1. J. - H. Ko, "Recent research trends in the development of new light sources for the backlight unit of liquid crystal display," Asian J. Phys. 14, 231-237 (2005).

2. J. H. Lee, Y. H. Ju, J.-H. Park, J.-Y. Lee, K.-B. Nahm, J. - H. Ko, and J. H. Kim, "Optical simulation study on the effect of reflecting properties of reflection films on the performances of collimating films for the LCD backlight applications," J. Information Display 9, 26-32 (2008).

3. M. F. Weber, C. A. Stover, L. R. Gilbert, T. J. Nevitt, and A. J. Ouderkirt, "Giant birefringent optics in multilayer polymer mirrors," Science 287, 2451-2456 (2000).

4. M. - Y. Yu, J. - H. Park, and J. - H. Ko, "Study on the luminance properties of optical films for flat-lamp-based backlights," J. Opt. Soc. Korea 12, pp. 174-177 (2008).

5. G. J. Park, T. S. Aum, D. H. Lee, J. H. Kwon, M. H. Lee, B. K. Jung, and H. S. Soh, "Enhancement of luminance and view angle by optimizing the structure of prism sheet and the diffusivity of reflective polarizer film," in Proc. IDW '06 Tech. Digest (Otsu Prince Hotel, Japan, Dec. 2006), pp. 953-956.

6. J. Jeong, J. Kim, M. Shin, M. Lee, J. Chung, H. Jeong, J. Kim, J. Kang, B. Hong, and G. Cho, "Luminance normalization of optical sheets in a backlight unit for LCD-TVs,” J. Korean Vac. Soc. 16, 424-243 (2007).

7. J.-H. Park and J. - H. Ko, "Optimization of the emitting structure of flat fluorescent lamps for LCD backlight applications," J. Opt. Soc. Korea 11, 118-123 (2007).

8. M.-Y. Yu, Y.-Y. Kim, J.-H. Ko, S.-H. Yu, and S.-E. Lee, "Optical characteristics of direct-lit flat-lamp backlights for LCD applications," in Proc. IDW '08 Tech. Digest (Niigata Convention Center, Japan, 2008), pp. 1803-1806.

9. G. R. Fowles, Introduction to Modern Optics, $2^{\text {nd }}$ ed. (Holt, Rinehart and Winston, New York, USA, 1975), pp. $38-45$.

10. P. Watson and G. T. Boyd, "Backlighting of mobile displays," in Mobile Displays, edited by A. K. Bhowmik, Z. Li, and P. J. Bos, (John Wiley \& Sons, The Atrium, Chichester, UK, 2008), pp. 219-223. 